\title{
Uniform confidence bands for functions estimated nonparametrically with instrumental variables
}

\author{
Joel L. Horowitz \\ Sokbae Lee
}

The Institute for Fiscal Studies

Department of Economics, UCL

cemmap working paper CWP18/09 


\title{
UNIFORM CONFIDENCE BANDS FOR FUNCTIONS ESTIMATED NONPARAMETRICALLY WITH INSTRUMENTAL VARIABLES
}

\author{
JOEL L. HOROWITZ AND SOKBAE LEE
}

\begin{abstract}
This paper is concerned with developing uniform confidence bands for functions estimated nonparametrically with instrumental variables. We show that a sieve nonparametric instrumental variables estimator is pointwise asymptotically normally distributed. The asymptotic normality result holds in both mildly and severely ill-posed cases. We present an interpolation method to obtain a uniform confidence band and show that the bootstrap can be used to obtain the required critical values. Monte Carlo experiments illustrate the finite-sample performance of the uniform confidence band.
\end{abstract}

JEL Classification Codes: C13, C14.

Key words: Bootstrap, instrumental variables, sieve estimator, uniform confidence band.

\section{INTRODUCTION}

This paper is concerned with developing a uniform confidence band for the unknown function $g$ in the model

$$
Y=g(X)+U ; E(U \mid W=w)=0 \text { for almost every } w,
$$

where $Y$ is a scalar dependent variable, $X \in \mathbb{R}^{q}$ is a continuously distributed explanatory variable that may be endogenous (that is, we allow the possibility that $E(U \mid X=x) \neq 0), W \in \mathbb{R}^{q}$ is a continuously distributed instrument for $X$, and $U$ is an unobserved random variable. The unknown function $g$ is nonparametric. It is assumed to satisfy mild regularity conditions but does not belong to a known, finite-dimensional parametric family. The data are an independent

Date: 28 July 2009.

The work of both authors was supported in part by the Economic and Social Research Council through its funding of the ESRC Centre for Microdata Methods and Practice (RES-589-28-0001). The research of Joel L. Horowitz was supported in part by NSF grant SES-0817552 and the research of Sokbae Lee was supported in part by ESRC research grant RES-000-22-2761. We would like to thank Xiaohong Chen for helpful comments. 
random sample $\left\{\left(Y_{i}, X_{i}, W_{i}\right): i=1, \ldots, n\right\}$ from the distribution of $(Y, X, W)$.

Nonparametric estimators of $g$ in (1.1) have been developed by Newey and Powell (2003); Hall and Horowitz (2005); Darolles, Florens, and Renault (2006); and Blundell, Chen, and Kristensen, (2007). Horowitz (2007) gave conditions for asymptotic normality of the kernel estimator of Hall and Horowitz (2005). Newey, Powell, and Vella (1999) presented a control function approach to estimating $g$ in a model that is different from (1.1) but allows endogeneity of $X$ and achieves identification through an instrument. The control function model is non-nested with (1.1) and is not discussed further in this paper. Chernozhukov, Imbens, and Newey (2007); Horowitz and Lee (2007); and Chernozhukov, Gagliardini, and Scaillet (2008) have developed methods for estimating a quantile-regression version of model (1.1). In the quantile regression, the condition $E(U \mid W=w)=0$ is replaced by

$$
P(U \leq 0 \mid W=w)=\alpha \quad \text { for some } \alpha \in(0,1) .
$$

Chen and Pouzo $(2008,2009)$ developed a method for estimating a large class of nonparametric and semiparametric conditional moment models with possibly non-smooth moments. This class includes (1.2).

This paper obtains asymptotic uniform confidence bands for $g$ in (1.1) by using a modified version of the sieve estimator of Blundell, Chen, and Kristensen (2007). Sieve estimators of $g$ are easier to compute than kernel-based estimators such as those of Darolles, Florens, and Renault (2006) and Hall and Horowitz (2005). Moreover, sieve estimators achieve the fastest possible rate of convergence under conditions that are weaker in important ways than those required by existing kernel-based estimators. The sieve estimator used in this paper was proposed by Horowitz (2008) in connection with a specification test for model (1.1). Here, we show that this estimator is pointwise asymptotically normal and that the bootstrap can be used to obtain simultaneous pointwise confidence intervals for $g\left(x_{1}\right), \ldots, g\left(x_{L}\right)$ on almost every finite grid of points $x_{1}, \ldots, x_{L}$. We obtain a uniform confidence band by using properties of $g$ such as smoothness or monotonicity to interpolate between the grid points. Hall and Titterington (1988) used interpolation to obtain uniform confidence bands for nonparametrically estimated probability density and conditional mean functions.

A seemingly natural approach to constructing a uniform confidence band is to obtain the asymptotic distribution of a suitably scaled version of $\sup _{x}|\hat{g}(x)-g(x)|$, where $\hat{g}$ is the estimator of $g$. However, when $\hat{g}$ is a sieve estimator, this is a difficult problem that has been 
solved only for special cases in which $g$ is a conditional mean function and certain restrictive conditions hold (Zhou, Shen, and Wolfe 1998; Wang and Yang 2009). Our interpolation approach avoids this problem. The resulting uniform confidence band is not asymptotically exact; its true and nominal coverage probabilities are not necessarily equal even asymptotically. But the confidence band can be made arbitrarily accurate (that is, the difference between the true and nominal asymptotic coverage probabilities can be made arbitrarily small) by making the grid $x_{1}, \ldots, x_{L}$ sufficiently fine. In practice, a confidence band can be computed at only finitely many points, so it makes little practical difference whether the confidence interval at each point is based on a finite-dimensional distribution or the distribution of a scaled version of $\sup _{x}|\hat{g}(x)-g(x)|$.

The remainder of the paper is organized as follows. Section 2 presents the sieve nonparametric IV estimator. Section 3 gives conditions under which the estimators of $g\left(x_{1}\right), \ldots, g\left(x_{L}\right)$ are asymptotically multivariate normally distributed when $X$ and $W$ are scalar random variables. Section 4 uses the results of Section 3 to obtain a uniform confidence band for $g$ when $X$ and $W$ are scalars. Section 5 establishes consistency of the bootstrap for estimating the confidence band. Section 6 extends the results of Sections 3.5 to the case in which $X$ and $W$ are random vectors. Section 7 reports the results of a Monte Carlo investigation of the finite-sample coverage probabilities of the uniform confidence bands, and concluding comments are given in Section 8. The proofs of theorems are in the appendix.

\section{The Sieve Nonparametric Estimator}

This section describes Horowitz's (2008) sieve estimator of $g$ when $X$ and $W$ are scalar random variables. Let $f_{W}$ denote the probability density function of $W, f_{X W}$ denote the probability density function of $(X, W)$, and

$$
m(w):=E(Y \mid W=w) f_{W}(w) .
$$

Assume, without loss of generality, that the support of $(X, W)$ is $[0,1]^{2}$. Define the operator $A$ by

$$
(A v)(w):=\int_{0}^{1} v(x) f_{X W}(x, w) d x .
$$

Then $g$ in 1.1 satisfies

$$
A g=m \text {. }
$$


For a function $v:[0,1] \mapsto \mathbb{R}$ and integer $l \geq 0$, define

$$
D_{l} v(x):=\frac{\partial^{l} v(x)}{\partial x^{l}}
$$

whenever the derivative exists, with the convention $D_{0} v(x)=v(x)$. Given an integer $s>0$, define the Sobolev norm

$$
\|v\|_{s}:=\left\{\sum_{l=0}^{s} \int_{0}^{1}\left[D_{l} v(x)\right]^{2} d x\right\}^{1 / 2}
$$

and the function space

$$
\mathcal{H}_{s}:=\left\{v:[0,1] \mapsto \mathbb{R}:\|v\|_{s} \leq C_{g}\right\}
$$

where $C_{g}<\infty$ is a constant. Assume that $g \in \mathcal{H}_{s}$ for some $s>0$ and that $\|g\|_{s}<C_{g}$.

The estimator of $g$ is defined in terms of series expansions of $g, m$, and $A$. Let $\left\{\psi_{j}: j=1,2, \ldots\right\}$ be a complete, orthonormal basis for $L_{2}[0,1]$. The expansions are

$$
\begin{aligned}
g(x) & =\sum_{j=1}^{\infty} b_{j} \psi_{j}(x), \\
m(w) & =\sum_{k=1}^{\infty} a_{k} \psi_{k}(w), \\
f_{X W}(x, w) & =\sum_{j=1}^{\infty} \sum_{k=1}^{\infty} c_{j k} \psi_{j}(x) \psi_{k}(w),
\end{aligned}
$$

where

$$
\begin{aligned}
b_{j} & =\int_{0}^{1} g(x) \psi_{j}(x) d x, \\
a_{k} & =\int_{0}^{1} m(w) \psi_{k}(w) d w \\
c_{j k} & =\int_{[0,1]^{2}} f_{X W}(x, w) \psi_{j}(x) \psi_{k}(w) d w d x .
\end{aligned}
$$


To estimate $g$, we need to estimate $a_{k}, m, c_{j k}$, and $f_{X W}$. The estimators are

$$
\begin{aligned}
\hat{a}_{k} & =n^{-1} \sum_{i=1}^{n} Y_{i} \psi_{k}\left(W_{i}\right), \\
\hat{m} & =\sum_{j=1}^{J_{n}} \hat{a}_{j} \psi_{j}, \\
\hat{c}_{j k} & =n^{-1} \sum_{1=1}^{n} \psi_{j}\left(X_{i}\right) \psi_{k}\left(W_{i}\right),
\end{aligned}
$$

and

$$
\hat{f}_{X W}(x, w)=\sum_{j=1}^{J_{n}} \sum_{k=1}^{J_{n}} \hat{c}_{j k} \psi_{j}(x) \psi_{k}(w),
$$

respectively, where $J_{n}<\infty$ is the series truncation point. Define the operator $\hat{A}_{n}$ that estimates $A$ by

$$
\left(\hat{A}_{n} v\right)(w):=\int_{0}^{1} v(x) \hat{f}_{X W}(x, w) d x .
$$

Define the subset of $\mathcal{H}_{s}$ :

$$
\mathcal{H}_{n s}:=\left\{v=\sum_{j=1}^{J_{n}} v_{j} \psi_{j}:\|v\|_{s} \leq C_{g}\right\} .
$$

The sieve estimator of $g$ is defined as

$$
\hat{g}_{n}:=\arg \min _{v \in \mathcal{H}_{n s}}\left\|\hat{A}_{n} v-\hat{m}\right\|,
$$

where $\|\cdot\|$ is the $L_{2}$ norm on $L_{2}[0,1]$. Under the assumptions of Section 3. $P\left(\hat{A}_{n} g_{n}=\hat{m}\right) \rightarrow 1$ as $n \rightarrow \infty$. Therefore,

$$
\hat{g}_{n}=\hat{A}_{n}^{-1} \hat{m}
$$

with probability approaching 1 as $n \rightarrow \infty$.

\section{Asymptotic Normality}

This section gives conditions under which $\hat{g}_{n}(x)$ is asymptotically normally distributed. Proving asymptotic normality of an estimator usually requires assumptions that are stronger than those needed for consistency or convergence at the asymptotically optimal rate. The assumptions made here are stronger than those used by Blundell, Chen, and Kristensen (2007) and Horowitz (2008) to prove that their estimators are consistent with the optimal rate of convergence. 
Define $A^{*}$ to be the adjoint operator of $A$ and

$$
\rho_{n}:=\sup _{h \in \mathcal{H}_{n s}:\|h\| \neq 0} \frac{\|h\|}{\left\|\left(A^{*} A\right)^{1 / 2} h\right\|},
$$

Blundell, Chen, and Kristensen (2007) call this the sieve measure of ill-posedness and discuss its relation to the eigenvalues of $A^{*} A$. Under suitable conditions, $\rho_{n}=O\left(J_{n}^{r}\right)$ if the eigenvalues, sorted in decreasing order, converge to zero at the rate $J_{n}^{-2 r}$ (mildly ill-posed case). If the eigenvalues converge exponentially fast (severely ill-posed case), then $\rho_{n}$ is proportional to $\exp \left(c J_{n}\right)$ for some finite $c>0$.

Assumption 3.1. (1) The support of $(X, W)$ is $[0,1]^{2}$. (2) $g \in \mathcal{H}_{s}$ and $\|g\|_{s}<C_{g}$ for some integer $s>0$ and finite constant $C_{g}$. (3) The operator $A$ is nonsingular. (4) $(X, W)$ has a probability density function $f_{X W}$ with respect to Lebesgue measure. In addition, $f_{X W}$ has $r \geq s$ bounded derivatives with respect to any combination of its arguments. (5) $\sup _{w \in[0,1]} E\left(Y^{2} \mid W=w\right) \leq C_{Y}$ for some $C_{Y}<\infty$.

Assumption 3.2. (1) The set of functions $\left\{\psi_{j}: j=1,2, \ldots\right\}$ is a complete, orthonormal basis for $L_{2}[0,1]$. (2) $\left\|g-\sum_{j=1}^{J} b_{j} \psi_{j}\right\|=O\left(J^{-s}\right)$.

Among other things, Assumptions 3.1 and 3.2 ensure that $f_{X W}$ is at least as smooth as $g$. Moreover, $A$ and $A^{*}$ map $L_{2}[0,1]$ into $\mathcal{H}_{s}$. Assumption 3.2 (2) is satisfied by a variety of bases including trigonometric functions, orthogonal polynomials, and splines.

Let $A_{n}$ be the operator on $L_{2}[0,1]$ whose kernel is

$$
a_{n}(x, w)=\sum_{j=1}^{J_{n}} \sum_{k=1}^{J_{n}} c_{j k} \psi_{j}(x) \psi_{k}(w) .
$$

Let $A_{n}^{*}$ denote the adjoint operator of $A_{n}$.

Assumption 3.3. The ranges of $A_{n}$ and $A_{n}^{*}$ are contained in $\mathcal{H}_{n s}$ for all sufficiently large $n$. Moreover

$$
\rho_{n} \sup _{h \in \mathcal{H}_{n s}}\left\|\left(A_{n}-A\right) h\right\|=O\left(J_{n}^{-s}\right) .
$$

Assumption 3.3 ensures that $A_{n}$ is a "sufficiently accurate" approximation to $A$. Condition (3.2) can be interpreted as a smoothness restriction on $f_{X W}$ or as a restriction on the sizes of the values of $c_{j k}$ for $j \neq k$. Condition (3.2) is satisfied automatically if $c_{j k}=c_{j j} \delta_{j k}$, where $\delta_{j k}$ is the Kronecker delta. Hall and Horowitz (2005) used a similar diagonality condition in their nonparametric instrumental variables estimator. 
Assumption 3.4. (1) $J_{n}^{-s}=o\left[\rho_{n}\left(J_{n} / n\right)^{1 / 2}\right]$. (2) $\left(\rho_{n} J_{n}\right) / n^{1 / 2} \rightarrow 0$.

Assumption 3.4 (1) requires $\hat{g}_{n}$ to be undersmoothed. That is, as $n \rightarrow \infty, J_{n}$ increases at a rate that is faster than the asymptotically optimal rate. As with other nonparametric estimators, undersmoothing ensures that the asymptotic bias of $\hat{g}_{n}$ is negligible. Assumption 3.4 (2) ensures that the asymptotic variance of $\hat{g}_{n}$ converges to zero.

Remark 1. (1) If $\rho_{n}=O\left(J_{n}^{r}\right)$ for some finite $r>0$, then we can set $J_{n} \propto n^{\eta}$, where $\frac{1}{2 r+2 s+1}<\eta<\frac{1}{2 r+2}$. if

(2) If $\rho_{n}=\exp \left(c J_{n}\right)$ for some finite $c>0$, Assumption 3.4 is satisfied

$$
J_{n}=\frac{\log n}{2 c}-\frac{2 s \alpha_{0}+1}{2 c} \log \log n
$$

for some $\alpha_{0}$ satisfying $0<\alpha_{0}<1$. The rate of increase must be logarithmic, and the constant multiplying $\log n$ must be $1 /(2 c)$. If the constant is larger, the integrated variance of $\hat{g}_{n}-g$ does not converge to 0 . If the constant is smaller, the bias dominates the variance. The higher order component of $J_{n}$ is important. If it is 0 or too small, the integrated variance does not converge to 0 . These requirements illustrate the delicacy of estimation in the severely ill-posed case.

Now define

$$
\begin{aligned}
& \delta_{n}(x, Y, X, W):= \\
& \sum_{k=1}^{J_{n}}\left\{\left[Y \psi_{k}(W)-a_{k}\right]-\sum_{j=1}^{J_{n}} b_{j}\left[\psi_{j}(X) \psi_{k}(W)-c_{j k}\right]\right\}\left(A_{n}^{-1} \psi_{k}\right)(x) .
\end{aligned}
$$

Also, define

$$
\sigma_{n}^{2}(x):=n^{-1} \operatorname{Var}\left[\delta_{n}(x, Y, X, W)\right] .
$$

Define $c_{n} \asymp d_{n}$ for any positive sequences of constants $c_{n}$ and $d_{n}$ to mean that $c_{n} / d_{n}$ is bounded away from 0 and $\infty$.

Assumption 3.5. For any $x \in[0,1], \sigma_{n}(x) \asymp\left\|\sigma_{n}\right\|$ except, possibly, if $x$ belongs to a set of Lebesgue measure 0 .

This condition is similar to Assumption 6 of Horowitz (2007). It rules out a form of superefficiency in which $g_{n}(x)-g(x)$ converges to 0 more rapidly than $\left\|g_{n}-g\right\|$.

Assumption 3.6. There exist constants $C<\infty$ and $v>0$ such that

$$
E_{Y X W}\left[\left|\delta_{n}(x, Y, X, W)\right|^{2+v}\right] \leq C
$$

for all sufficiently large $n$ and for all $x \in[0,1]$. 
By a triangular-array version of the weak law of large numbers, e.g. Theorem 2 of Andrews (1988), Assumption 3.6 implies that as $n \rightarrow \infty$,

$$
n^{-1} \sum_{i=1}^{n} \delta_{n}\left(x, Y_{i}, X_{i}, W_{i}\right)^{2} \rightarrow{ }_{p} E_{Y X W}\left[\delta_{n}(x, Y, X, W)^{2}\right] .
$$

Assumption 3.6 also ensures that we can apply a triangular-version of the Lindeberg-Levy central limit theorem.

Let $\left\{x_{1}, \ldots, x_{L}\right\}$ denote a set of $L$ points in $[0,1]$. The following theorem establishes the joint asymptotic normality of the sieve estimator of $\hat{g}_{n}\left(x_{1}\right), \ldots, \hat{g}_{n}\left(x_{L}\right)$.

Theorem 3.1. Let Assumptions 3.1]3.6 hold. Then as $n \rightarrow \infty$,

$$
\left\{\frac{\left[\hat{g}_{n}\left(x_{1}\right)-g\left(x_{1}\right)\right]}{\sigma_{n}\left(x_{1}\right)}, \ldots, \frac{\left[\hat{g}_{n}\left(x_{L}\right)-g\left(x_{L}\right)\right]}{\sigma_{n}\left(x_{L}\right)}\right\} \rightarrow_{d} \mathbf{N}\left[0, V_{g}\left(x_{1}, \ldots, x_{L}\right)\right],
$$

except, possibly, if $x_{1}, \ldots, x_{L}$ belong to a set of Lebesgue measure 0 in $[0,1]^{L}$, where $V_{g}\left(x_{1}, \ldots, x_{L}\right)$ is the $L \times L$ matrix whose $(j, k)$ element is

$$
V_{j k}:=E\left[\frac{\delta_{n}\left(x_{j}, Y, X, W\right) \delta_{n}\left(x_{k}, Y, X, W\right)}{\left(\operatorname{Var}\left[\delta_{n}\left(x_{j}, Y, X, W\right)\right]\right)^{1 / 2}\left(\operatorname{Var}\left[\delta_{n}\left(x_{k}, Y, X, W\right)\right]\right)^{1 / 2}}\right] .
$$

3.1. Estimation of $\sigma_{n}^{2}(x)$. To make use of the asymptotic results obtained in Theorem 3.1, it is necessary to estimate $\sigma_{n}^{2}(x)$. To do this, let

$$
\tilde{\delta}_{n}^{*}(x, Y, X, W):=\left[Y-\hat{g}_{n}(X)\right] \sum_{k=1}^{J_{n}} \psi_{k}(W) \psi_{k}(x) .
$$

Then $\sigma_{n}^{2}(x)$ can be estimated consistently by

$$
s_{n}^{2}(x):=n^{-2} \sum_{i=1}^{n}\left\{\hat{A}_{n}^{-1}\left[\tilde{\delta}_{n}^{*}\left(x, Y_{i}, X_{i}, W_{i}\right)-\bar{\delta}_{n}^{*}(x)\right]\right\}^{2},
$$

where

$$
\bar{\delta}_{n}^{*}(x):=n^{-1} \sum_{i=1}^{n} \tilde{\delta}_{n}^{*}\left(x, Y_{i}, X_{i}, W_{i}\right)
$$

We now state the consistency of $s_{n}^{2}(x)$.

Theorem 3.2. Let Assumptions 3.1.3.6 hold. Then as $n \rightarrow \infty$,

$$
\frac{s_{n}^{2}(x)}{\sigma_{n}^{2}(x)} \rightarrow_{p} 1 .
$$




\section{Uniform Confidence Band}

The results in Section 3 make it possible to form joint confidence intervals and, by interpolation, a uniform confidence band for $g$ over $[a, b]$ for constants $a$ and $b$ such that $0 \leq a<b \leq 1$. To form joint confidence intervals, let $\left\{x_{1}, \ldots, x_{L}\right\}$ be points sampled from uniform distributions on the intervals $[a, a+(b-a) / L),[a+(b-a) / L, a+2(b-$ $a) / L), \ldots,[a+(L-1)(b-a) / L, b]$. Random sampling this way avoids exceptional sets of Lebesgue measure 0 in Theorem 3.1. Let $z_{\alpha}$ satisfy

$$
P\left[\sup _{1 \leq l \leq L}\left|\mathbb{Z}_{l}\right|>z_{\alpha}\right]=\alpha,
$$

where $\mathbb{Z}_{l}$ is the $l$-th component of $\mathbb{Z} \sim \mathbf{N}\left[0, V_{g}\left(x_{1}, \ldots, x_{L}\right)\right]$. Then

$$
\hat{g}\left(x_{l}\right)-z_{\alpha} s_{n}\left(x_{l}\right) \leq g\left(x_{l}\right) \leq \hat{g}\left(x_{l}\right)+z_{\alpha} s_{n}\left(x_{l}\right)
$$

are joint asymptotic $100(1-\alpha) \%$ confidence intervals for $g\left(x_{1}\right), \ldots, g\left(x_{L}\right)$, $l=1, \ldots, L$. We now describe two ways of obtaining a uniform confidence band for $g$ by interpolating the joint confidence intervals. A method for estimating $z_{\alpha}$ is described in Section 5 .

\subsection{A Uniform Confidence Band under Piecewise Monotonic-}

ity. In this subsection, assume that $g$ is monotonic on each of the grid intervals. This is reasonable if $L$ is sufficiently large. Let

$$
\bar{x}_{l}:=\operatorname{argmax}\left\{\hat{g}\left(x_{l}\right)+z_{\alpha} s_{n}\left(x_{l}\right), \hat{g}\left(x_{l+1}\right)+z_{\alpha} s_{n}\left(x_{l+1}\right)\right\},
$$

and

$$
\underline{x}_{l}:=\operatorname{argmin}\left\{\hat{g}\left(x_{l}\right)-z_{\alpha} s_{n}\left(x_{l}\right), \hat{g}\left(x_{l+1}\right)-z_{\alpha} s_{n}\left(x_{l+1}\right)\right\} .
$$

Then by the assumed monotonicity of $g$ on $\left[x_{l}, x_{l+1}\right]$,

$$
\hat{g}\left(\underline{x}_{l}\right)-z_{\alpha} s_{n}\left(\underline{x_{l}}\right) \leq g(x) \leq \hat{g}\left(\bar{x}_{l}\right)+z_{\alpha} s_{n}\left(\bar{x}_{l}\right)
$$

uniformly over $x \in\left[x_{l}, x_{l+1}\right], l=1, \ldots, L-1$. Putting these intervals together gives a uniform confidence band for $g$ over $[a, b]$. The asymptotic coverage probability is at least $1-\alpha$ and it can be made arbitrarily close to $1-\alpha$ by making $L$ sufficiently large.

4.2. A Uniform Confidence Band under Lipschitz Continuity. Alternatively, assume that $g$ is Lipschitz continuous. That is,

$$
|g(x)-g(y)| \leq C_{L}|x-y|
$$

for some constant $C_{L}$ and any $x, y \in[a, b]$. For any $x \in[a+(b-$ $a) / L, a+(L-1)(b-a) / L]$, choose $l$ such that $\left|x-x_{l}\right|$ is minimized. 
First note that (4.1) is equivalent to

$$
\begin{aligned}
& \hat{g}\left(x_{l}\right)-z_{\alpha} s_{n}\left(x_{l}\right)+\left[g(x)-g\left(x_{l}\right)\right] \\
& \leq g(x) \leq \hat{g}\left(x_{l}\right)+z_{\alpha} s_{n}\left(x_{l}\right)+\left[g(x)-g\left(x_{l}\right)\right] .
\end{aligned}
$$

Then 4.2 implies

$$
\hat{g}\left(x_{l}\right)-z_{\alpha} s_{n}\left(x_{l}\right)-C_{L}\left|x-x_{l}\right| \leq g(x) \leq \hat{g}\left(x_{l}\right)+z_{\alpha} s_{n}\left(x_{l}\right)+C_{L}\left|x-x_{l}\right|,
$$

so that

$$
\hat{g}\left(x_{l}\right)-z_{\alpha} s_{n}\left(x_{l}\right)-\frac{C_{L}}{L} \leq g(x) \leq \hat{g}\left(x_{l}\right)+z_{\alpha} s_{n}\left(x_{l}\right)+\frac{C_{L}}{L}
$$

uniformly over $x \in\left[x_{l}-1 / L, x_{l}+1 / L\right]$. Putting these intervals in (4.3) together gives a uniform confidence band for $g$ over $[a, b]$. Again the asymptotic coverage probability exceeds $1-\alpha$ but can be made arbitrarily close to $1-\alpha$ by making $L$ sufficiently large.

\section{Bootstrap Estimation of $z_{\alpha}$}

This section shows that the bootstrap consistently estimates the joint asymptotic distribution of $\left[\hat{g}_{n}\left(x_{1}\right)-g\left(x_{1}\right)\right] / s_{n}\left(x_{1}\right), \ldots,\left[\hat{g}_{n}\left(x_{L}\right)-\right.$ $\left.g\left(x_{L}\right)\right] / s_{L}\left(x_{L}\right)$. It follows that the bootstrap consistently estimates the critical value $z_{\alpha}$ in 4.1 .

It is shown in the proof of theorem 3.1 that the leading term of the asymptotic expansion of $\hat{g}_{n}(x)-g(x)$ is

$$
S_{n}(x)=n^{-1} \sum_{i=1}^{n} \delta_{n}\left(x, Y_{i}, X_{i}, W_{i}\right),
$$

where $\delta_{n}(x, Y, X, W)$ is defined in (3.3). Therefore, it suffices to show that the bootstrap consistently estimates the asymptotic distribution of $t_{n}\left(x_{1}\right), \ldots, t_{n}\left(x_{L}\right)$, where $t_{n}(x):=S_{n}(x) / s_{n}(x)$. Define $g_{n}(x):=$ $\sum_{j=1}^{J_{n}} b_{j} \psi_{j}(x)$ for any $x \in[0,1]$. Define

$$
\tilde{S}_{n}(x):=n^{-1} A_{n}^{-1} \sum_{i=1}^{n} \tilde{\delta}_{n}\left(x, Y_{i}, X_{i}, W_{i}\right),
$$

where

$$
\tilde{\delta}_{n}(x, Y, X, W):=\left[Y-g_{n}(X)\right] \sum_{k=1}^{J_{n}} \psi_{k}(W) \psi_{k}(x) .
$$

Then $S_{n}(x)$ can be rewritten as

$$
S_{n}(x)=\tilde{S}_{n}(x)-E \tilde{S}_{n}(x) .
$$


Define $\tilde{t}_{n}(x)=\left[\tilde{S}_{n}(x)-E \tilde{S}_{n}(x)\right] / s_{n}(x)$. We now describe a bootstrap procedure that consistently estimates the asymptotic distribution of $\tilde{t}_{n}\left(x_{1}\right), \ldots, \tilde{t}_{n}\left(x_{L}\right)$.

Let $\left\{\left(Y_{i}^{*}, X_{i}^{*}, W_{i}^{*}\right): i=1, \ldots, n\right\}$ denote a bootstrap sample that is obtained by sampling the data $\left\{\left(Y_{i}, X_{i}, W_{i}\right): i=1, \ldots, n\right\}$ randomly with replacement. The bootstrap version of $\tilde{S}_{n}(x)$ is

$$
\tilde{S}_{n}^{*}(x):=n^{-1} \hat{A}_{n}^{-1} \sum_{i=1}^{n} \tilde{\delta}_{n}^{*}\left(x, Y_{i}^{*}, X_{i}^{*}, W_{i}^{*}\right),
$$

where $\tilde{\delta}_{n}^{*}(x, Y, X, W)$ is defined in 3.5). A bootstrap version of $t_{n}(x)$ is

$$
t_{n}^{*}(x):=\left[\tilde{S}_{n}^{*}(x)-\hat{A}_{n}^{-1} \bar{\delta}_{n}^{*}(x)\right] / s_{n}(x),
$$

where $\bar{\delta}_{n}^{*}(x)$ is defined in (3.7). The $\alpha$-level bootstrap critical value, $z_{\alpha}^{*}$, estimates $z_{\alpha}$ in (4.1) and can be obtained as the solution to

$$
P^{*}\left[\sup _{1 \leq l \leq L}\left|t_{n}^{*}\left(x_{l}\right)\right|>z_{\alpha}^{*}\right]=\alpha
$$

where $P^{*}$ denotes the probability measure induced by bootstrap sampling conditional on the data $\left\{\left(Y_{i}, X_{i}, W_{i}\right): i=1, \ldots, n\right\}$. One nice feature of the bootstrap procedure is that it is unnecessary to estimate $V_{g}\left(x_{1}, \ldots, x_{L}\right)$.

An alternative bootstrap version of $t_{n}(x)$ is

$$
t_{n}^{* *}(x):=\left[\tilde{S}_{n}^{*}(x)-\hat{A}_{n}^{-1} \bar{\delta}_{n}^{*}(x)\right] / s_{n}^{*}(x),
$$

where $s_{n}^{*}(x)$ is the bootstrap analog of $s_{n}(x)$. Specifically,

$$
s_{n}^{*}(x):=\left[n^{-2} \sum_{i=1}^{n}\left\{\left(\hat{A}_{n}^{*}\right)^{-1}\left[\tilde{\delta}_{n}^{* *}\left(x, Y_{i}^{*}, X_{i}^{*}, W_{i}^{*}\right)-\bar{\delta}_{n}^{* *}(x)\right]\right\}^{2}\right]^{1 / 2},
$$

where $\hat{A}_{n}^{*}$ and $\hat{g}_{n}^{*}$, respectively, are the same as $\hat{A}_{n}$ and $\hat{g}_{n}$ in 2.3 and (2.4), but with the bootstrap sample $\left\{\left(Y_{i}^{*}, X_{i}^{*}, W_{i}^{*}\right): i=1, \ldots, n\right\}$ in place of the estimation data,

$$
\tilde{\delta}_{n}^{* *}\left(x, Y_{i}^{*}, X_{i}^{*}, W_{i}^{*}\right):=\left[Y_{i}^{*}-\hat{g}_{n}^{*}\left(X_{i}^{*}\right)\right] \sum_{k=1}^{J_{n}} \psi_{k}\left(W_{i}^{*}\right) \psi_{k}(x) .
$$

and

$$
\bar{\delta}_{n}^{* *}(x):=n^{-1} \sum_{i=1}^{n} \tilde{\delta}_{n}^{* *}\left(x, Y_{i}^{*}, X_{i}^{*}, W_{i}^{*}\right)
$$


Let $\mathcal{L}^{*}(\ldots)$ denote the conditional distribution $L\left(\ldots \mid\left\{\left(Y_{i}, X_{i}, W_{i}\right)\right.\right.$ : $i=1, \ldots, n\})$ and let $d_{\infty}\left(H_{1}, H_{2}\right)$ denote the Kolmogorov distance, that is the sup norm between two distribution functions $H_{1}$ and $H_{2}$. The following theorem establishes the consistency of the bootstrap and implies that $z_{\alpha}^{*}$ is a consistent estimator of $z_{\alpha}$.

Theorem 5.1. Let Assumptions 3.1 3.6 hold. Then as $n \rightarrow \infty$,

$$
d_{\infty}\left(\mathcal{L}^{*}\left\{t_{n}^{*}\left(x_{1}\right), \ldots, t_{n}^{*}\left(x_{L}\right)\right\}, \mathbf{N}\left[0, V_{g}\left(x_{1}, \ldots, x_{L}\right)\right]\right) \rightarrow 0 \text { in probability }
$$

and

$d_{\infty}\left(\mathcal{L}^{*}\left\{t_{n}^{* *}\left(x_{1}\right), \ldots, t_{n}^{* *}\left(x_{L}\right)\right\}, \mathbf{N}\left[0, V_{g}\left(x_{1}, \ldots, x_{L}\right)\right]\right) \rightarrow 0$ in probability.

\section{Multivariate Model}

This section extends the results of Sections 2,5 to a multivariate model in which $X$ and $W$ are $q$-dimensional random vectors. Assume that the support of $(X, W)$ contained is $[0,1]^{2 q}$. Let $\left\{\psi_{j}: j=1,2, \ldots\right\}$ be a complete, orthonormal basis for $L_{2}[0,1]^{q}$. Define the operator $A$ by

$$
(A v)(w):=\int_{[0,1]^{q}} v(x) f_{X W}(x, w) d x .
$$

As in Section 2, the estimator of $g$ is defined in terms of series expansions of $g, m$, and $A$. The expansions are like those in 2.1 with the following generalized Fourier coefficients:

$$
\begin{aligned}
b_{j} & =\int_{[0,1]^{q}} g(x) \psi_{j}(x) d x, \\
a_{k} & =\int_{[0,1]^{q}} m(w) \psi_{j}(w) d w, \\
c_{j k} & =\int_{[0,1]^{2 q}} f_{X W}(x, w) \psi_{j}(x) \psi_{k}(w) d w d x .
\end{aligned}
$$

The estimators of $a_{k}, m, c_{j k}$, and $f_{X W}$ are the same as in (2.2), but with the basis functions for $L_{2}[0,1]^{q}$. Also, define the operator $\hat{A}_{n}$ that estimates $A$ by

$$
\left(\hat{A}_{n} v\right)(w):=\int_{[0,1]^{q}} v(x) \hat{f}_{X W}(x, w) d x .
$$

The sieve estimator of $g$ is as in (2.4), where $\|\cdot\|$ is now the norm on $L_{2}[0,1]^{q}$. Then the asymptotic normality result of Section 3 can be extended to the multivariate model with minor modifications. 
As in Section 4, it is possible to form joint confidence set for $g$ in the multivariate model. However, it is difficult to display joint confidence intervals or a uniform confidence set when $X$ is multidimensional. Therefore, we consider a one-dimensional projection of a joint confidence set for $g$.

Assume without loss of generality that the first component of $X$ is the direction of interest. Let $\left\{x_{11}, \ldots, x_{1 L}\right\}$ be points sampled from uniform distributions on the intervals $[a, a+(b-a) / L),[a+(b-a) / L, a+$ $2(b-a) / L), \ldots,[a+(L-1)(b-a) / L, b]$. Let $\sigma_{n}^{2}(x)$ denote a multivariate version of (3.4) and $s_{n}^{2}(x)$ denote a consistent estimator of $\sigma_{n}^{2}(x)$ as in (3.6). For a fixed value, say $x_{-1}$, of remaining components of $X$,

$\hat{g}\left(x_{1 l}, x_{-1}\right)-z_{\alpha} s_{n}\left(x_{1 l}, x_{-1}\right) \leq g\left(x_{1 l}, x_{-1}\right) \leq \hat{g}\left(x_{1 l}, x_{-1}\right)+z_{\alpha} s_{n}\left(x_{1 l}, x_{-1}\right)$

are joint asymptotic $100(1-\alpha) \%$ confidence intervals for $\left\{g\left(x_{1 l}\right): l=\right.$ $1, \ldots, L\}$ over $[a, b]$, where

$$
P\left[\sup _{1 \leq l \leq L}\left|\mathbb{Z}_{l}\right|>z_{\alpha}\right]=\alpha,
$$

and $\mathbb{Z}_{l}$ is the $l$-th component of $\mathbb{Z}$. Here, $\mathbb{Z}$ is the $L$-dimensional meanzero normal vector whose covariance matrix is the asymptotic covariance matrix of

$$
\left\{\frac{\left[\hat{g}_{n}\left(x_{11}, x_{-1}\right)-g\left(x_{11}, x_{-1}\right)\right]}{\sigma_{n}\left(x_{11}, x_{-1}\right)}, \ldots, \frac{\left[\hat{g}_{n}\left(x_{1 L}, x_{-1}\right)-g\left(x_{1 L}, x_{-1}\right)\right]}{\sigma_{n}\left(x_{1 L}, x_{-1}\right)}\right\} .
$$

We can construct the uniform confidence band of 6.2 as in Section 4 by assuming piecewise monotonicity or Lipschitz continuity. As in Section 5 , the critical value $z_{\alpha}$ can be obtained by the bootstrap.

\section{Monte Carlo Experiments}

This section reports the results of a Monte Carlo investigation of the coverage probabilities of the joint confidence intervals and uniform confidence bands using the bootstrap-based critical values of Section 5 .

As in Horowitz (2007), realizations of $(Y, X, W)$ were generated from the model

$$
\begin{aligned}
f_{X W}(x, w) & =C_{f} \sum_{j=1}^{\infty}(-1)^{j+1} j^{-\alpha / 2} \sin (j \pi x) \sin (j \pi w), \\
g(x) & =2.2 x, \\
Y & =E[g(X) \mid W]+V,
\end{aligned}
$$

where $C_{f}$ is a normalization constant chosen so that the integral of the joint density of $(X, W)$ equals one and $V \sim \mathbf{N}(0,0.01)$. Experiments 
were carried out with $\alpha=1.2$ and $\alpha=10$. The sample size is $n=200$. There are 1000 Monte Carlo replications in each experiment.

The grid $\left(x_{1}, \ldots, x_{L}\right)$ used to form joint confidence intervals and uniform confidence bands consists of 100 points. The Monte Carlo results are not sensitive to variations in the value of $L$ over the range 25 to 100 . The basis functions are Legendre polynomials that have had their supports shifted and have been normalized to make them orthonormal on $[0,1]$. The critical values are obtained by using the two bootstrap methods of Section 5 with 1000 bootstrap replications. The confidence bands were computed by using the piecewise monotonicity method of Section

4.1. The joint confidence intervals are for $\left(x_{1}, \ldots, x_{L}\right) \in[a, b]$ and the uniform confidence band is for any $x \in[a, b]=[0.2,0.8],[0.1,0.9]$ or $[0.01,0.99]$.

The results of the experiments are shown in Tables 1, 2, In each table, columns 3-5 show the empirical coverage probabilities of the joint confidence intervals, and columns 6-8 show the empirical coverage probabilities of the uniform confidence bands. We show the results of experiments with $J_{n}=3,4,5$, and 6 . The results show that the differences between the nominal and empirical coverage probabilities are small when the critical value is based on $t_{n}^{* *}(x)$ and $J_{n}=3$ or 4 .

\section{Conclusions}

This paper has given conditions under which a sieve nonparametric IV estimator is pointwise asymptotically normally distributed. The asymptotic normality result holds in both mildly and severely ill-posed cases. We have also shown that joint pointwise confidence intervals can be interpolated to obtain a uniform confidence band for the estimated function. The bootstrap can be used to estimate the critical values needed to form confidence intervals and bands. The results of Monte Carlo experiments show that the differences between nominal and empirical coverage probabilities are small when the critical values are obtained by using a suitable version of the bootstrap.

\section{Appendix A. Proofs}

We begin with the proof of Theorem 3.1. Because $\hat{g}_{n}=\hat{A}_{n}^{-1} \hat{m}$ with probability approaching 1 , it suffices to establish the asymptotic distribution of $\hat{h} \equiv \hat{A}_{n}^{-1} \hat{m}$.

Define

$$
m_{n}:=\sum_{k=1}^{J_{n}} a_{k} \psi_{k}
$$


Then

$$
A_{n} \hat{h}+\left(\hat{A}_{n}-A_{n}\right) \hat{h}=\hat{m}
$$

so that

$$
\begin{aligned}
\hat{h} & =A_{n}^{-1} \hat{m}-A_{n}^{-1}\left(\hat{A}_{n}-A_{n}\right) \hat{h} \\
& =A_{n}^{-1} \hat{m}-A_{n}^{-1}\left(\hat{A}_{n}-A_{n}\right) g-A_{n}^{-1}\left(\hat{A}_{n}-A_{n}\right)(\hat{h}-g) .
\end{aligned}
$$

Recall that $g_{n}=\sum_{j=1}^{J_{n}} b_{j} \psi_{j}$. Write

$$
A_{n}^{-1} \hat{m}-g=A_{n}^{-1}\left(\hat{m}-m_{n}\right)+\left(A_{n}^{-1} m_{n}-g_{n}\right)+\left(g_{n}-g\right) .
$$

Combining A.1 with A.2 yields $\hat{h}-g=S_{n}+R_{n}$, where

$$
S_{n}:=A_{n}^{-1}\left(\hat{m}-m_{n}\right)-A_{n}^{-1}\left(\hat{A}_{n}-A_{n}\right) g
$$

and $R_{n}:=R_{n 1}+R_{n 2}+R_{n 3}$ with

$$
\begin{aligned}
& R_{n 1}=-A_{n}^{-1}\left(\hat{A}_{n}-A_{n}\right)(\hat{h}-g), \\
& R_{n 2}=A_{n}^{-1} m_{n}-g_{n}, \\
& R_{n 3}=g_{n}-g .
\end{aligned}
$$

We now prove three lemmas that are useful to prove Theorem 3.1 .

Lemma A.1. We have that

$$
\left\|A_{n}^{-1}\right\| \leq O\left(\rho_{n}\right)
$$

Proof of Lemma A.1. First note that by Assumption 3.3, the eigenfunctions of $A_{n}^{*} A_{n}$ are in $\mathcal{H}_{s}$ for all sufficiently large $n$. Hence, since the dimension of $A_{n}^{*} A_{n}$ is $J_{n}$, we have that the eigenfunctions of $A_{n}^{*} A_{n}$ are in $\mathcal{H}_{n s}$ as well.

Now $\left\|A_{n}^{-1}\right\|^{2}$ is the largest eigenvalue of $\left(A_{n}^{-1}\right)^{*} A_{n}^{-1}=\left(A_{n} A_{n}^{*}\right)^{-1}$, which is the inverse of the smallest eigenvalue of $A_{n} A_{n}^{*}$ or, equivalently, the inverse of the smallest eigenvalue of $A_{n}^{*} A_{n}$. Since the smallest eigenvalue of $A_{n}^{*} A_{n}$ minimizes $\left\|A_{n}\right\|^{2}$, it suffices to the find the inverse of

But

$$
\inf _{h \in \mathcal{H}_{n s}} \frac{\left\|A_{n} h\right\|}{\|h\|}
$$

$$
\begin{aligned}
\rho_{n}^{-1} & =\inf _{h \in \mathcal{H}_{n s}} \frac{\|A h\|}{\|h\|}=\inf _{h \in \mathcal{H}_{n s}} \frac{\left\|A_{n} h+\left(A-A_{n}\right) h\right\|}{\|h\|} \\
& \leq \inf _{h \in \mathcal{H}_{n s}} \frac{\left\|A_{n} h\right\|+\left\|\left(A-A_{n}\right) h\right\|}{\|h\|} \\
& =\inf _{h \in \mathcal{H}_{n s}} \frac{\left\|A_{n} h\right\|}{\|h\|}+O\left(\rho_{n}^{-1} J_{n}^{-s}\right)
\end{aligned}
$$


by (3.2). Therefore,

$$
\inf _{h \in \mathcal{H}_{n s}} \frac{\left\|A_{n} h\right\|}{\|h\|} \geq \rho_{n}^{-1}+O\left(\rho_{n}^{-1} J_{n}^{-s}\right)=\rho_{n}^{-1}\left[1+O\left(J_{n}^{-s}\right)\right],
$$

which implies that

$$
\left\|A_{n}^{-1}\right\| \leq \rho_{n}\left[1+O\left(J_{n}^{-s}\right)\right] .
$$

This proves the lemma.

Lemma A.2. We have that

$$
\left\|R_{n 1}\right\|=O\left[\rho_{n}^{2}\left(J_{n} / n\right)\right] .
$$

Proof of Lemma A.2. By Horowtiz (2008),

$$
\begin{aligned}
\|\hat{h}-g\| & =O_{p}\left[J_{n}^{-s}+\rho_{n}\left(J_{n} / n\right)^{1 / 2}\right] \\
& =O_{p}\left[\rho_{n}\left(J_{n} / n\right)^{1 / 2}\right],
\end{aligned}
$$

where the last equality follows from undersmoothing (See Assumption 3.4 (1)). Note that by Lemma A.1.

$$
\begin{aligned}
\left\|R_{n 1}\right\| & =\left\|A_{n}^{-1}\right\|\left\|\left(\hat{A}_{n}-A_{n}\right)(\hat{h}-g)\right\| \\
& \leq O\left(\rho_{n}\right)\left\|\hat{A}_{n}-A_{n}\right\|\|\hat{h}-g\| \\
& =O\left(\rho_{n}\right) O_{p}\left[\left(J_{n} / n\right)^{1 / 2}\right]\|\hat{h}-g\|,
\end{aligned}
$$

which proves the lemma.

Lemma A.3. We have that

$$
\left\|R_{n 2}\right\|=O\left(J_{n}^{-s}\right) .
$$

Proof of Lemma A.3. Note that by Lemma A.1.

$$
\left\|R_{n 2}\right\| \leq\left\|A_{n}^{-1}\right\|\left\|m_{n}-A_{n} g_{n}\right\| \leq O\left(\rho_{n}\right)\left\|m_{n}-A_{n} g_{n}\right\| .
$$

Also, note that

$$
m_{n}-A_{n} g_{n}=\sum_{j=J_{n}+1}^{\infty} \sum_{k=1}^{J_{n}} b_{j} c_{j k} \psi_{k},
$$

and

$$
\left(A-A_{n}\right) g=\sum_{j=J_{n}+1}^{\infty} \sum_{k=1}^{J_{n}} b_{j} c_{j k} \psi_{k}+\sum_{j=1}^{\infty} \sum_{k=J_{n}+1}^{\infty} b_{j} c_{j k} \psi_{k} .
$$


Therefore,

$$
\left\|\left(A-A_{n}\right) g\right\|^{2}=\left\|m_{n}-A_{n} g_{n}\right\|^{2}+\sum_{k=J_{n}+1}^{\infty}\left(\sum_{j=1}^{\infty} b_{j} c_{j k}\right)^{2},
$$

which implies that

$$
\rho_{n}\left\|m_{n}-A_{n} g_{n}\right\| \leq \rho_{n}\left\|\left(A-A_{n}\right) g\right\| .
$$

Now note that Assumption 3.3 implies that

$$
\rho_{n} \sup _{h \in \mathcal{H}_{n s}}\left\|\left(A_{n}-A\right) h\right\|=O\left(J_{n}^{-s}\right) .
$$

Therefore, under A.3, we have that

$$
\begin{aligned}
\rho_{n}\left\|\left(A-A_{n}\right) g\right\| & \leq \rho_{n}\left\|\left(A-A_{n}\right) g_{n}\right\|+\rho_{n}\left\|\left(A-A_{n}\right)\left(g-g_{n}\right)\right\| \\
& =O\left(J_{n}^{-s}\right),
\end{aligned}
$$

since $\rho_{n}\left\|\left(A-A_{n}\right)\left(g-g_{n}\right)\right\|=o\left(J_{n}^{-s}\right)$. Therefore, we have proved the lemma.

Proof of Theorem 3.1. Note that by Assumption 3.2 $(2),\left\|R_{n 3}\right\|=O\left(J_{n}^{-s}\right)$. This is asymptotically negligible because of undersmoothing (Assumption 3.4 (1)). Therefore, by Lemmas A.2 and A.3 with the conditions on $J_{n}$ in Assumption 3.4 .

$$
\left\|R_{n}\right\|=o_{p}\left[\rho_{n}\left(J_{n} / n\right)^{1 / 2}\right] .
$$

Now using the series expansions, we have that

$$
\begin{aligned}
{\left[A_{n}^{-1}\left(\hat{m}-m_{n}\right)\right](x) } & =\sum_{k=1}^{J_{n}}\left[\hat{a}_{k}-a_{k}\right]\left(A_{n}^{-1} \psi_{k}\right)(x) \\
& =n^{-1} \sum_{i=1}^{n} \sum_{k=1}^{J_{n}}\left[Y_{i} \psi_{k}\left(W_{i}\right)-a_{k}\right]\left(A_{n}^{-1} \psi_{k}\right)(x)
\end{aligned}
$$

and

$$
\begin{aligned}
{\left[A_{n}^{-1}\left(\hat{A}_{n}-A_{n}\right) g\right](x) } & =\sum_{j=1}^{J_{n}} \sum_{k=1}^{J_{n}} b_{j}\left(\hat{c}_{j k}-c_{j k}\right)\left(A_{n}^{-1} \psi_{k}\right)(x) \\
& =n^{-1} \sum_{i=1}^{n} \sum_{j=1}^{J_{n}} \sum_{k=1}^{J_{n}} b_{j}\left[\psi_{j}\left(X_{i}\right) \psi_{k}\left(W_{i}\right)-c_{j k}\right]\left(A_{n}^{-1} \psi_{k}\right)(x) .
\end{aligned}
$$

Therefore,

$$
S_{n}(x)=n^{-1} \sum_{i=1}^{n} \delta_{n}\left(x, Y_{i}, X_{i}, W_{i}\right)
$$


A triangular-array version of the Lindeberg-Levy central theorem yields the result that

$$
\frac{S_{n}(x)}{\sigma_{n}(x)} \rightarrow_{d} \mathbf{N}(0,1)
$$

Now let $\left\{x_{1}, \ldots, x_{L}\right\}$ be a set of $L$ points in $[0,1]$. Then, the CramérWold device yields the result that

$$
\left\{\frac{S_{n}\left(x_{1}\right)}{\sigma_{n}\left(x_{l}\right)}, \ldots, \frac{S_{n}\left(x_{L}\right)}{\sigma_{n}\left(x_{L}\right)}\right\} \rightarrow_{d} \mathbf{N}\left[0, V_{g}\left(x_{1}, \ldots, x_{L}\right)\right] .
$$

Under the assumption $\sigma_{n}(x) \asymp\left\|\sigma_{n}\right\|$, the theorem follows if we can show that

$$
\left\|\sigma_{n}\right\|=O_{p}\left[\rho_{n}\left(J_{n} / n\right)^{1 / 2}\right] .
$$

To show A.5, write

$$
\begin{aligned}
\int_{0}^{1} \sigma_{n}^{2}(x) d x & =n^{-1} \int_{0}^{1} \operatorname{Var}\left[\delta_{n}(x, Y, X, W)\right] d x \\
& =E \int_{0}^{1}\left[S_{n}(x)\right]^{2} d x \\
& =E\left\|S_{n}\right\|^{2} \\
& \leq 2 E\left\|A_{n}^{-1} A_{n}\left(\hat{m}-m_{n}\right)\right\|^{2}+2 E\left\|A_{n}^{-1} A_{n}\left(\hat{A}_{n}-A_{n}\right) g\right\|^{2} \\
& \leq 2\left\|A_{n}^{-1}\right\|^{2}\left[E\left\|A_{n}\left(\hat{m}-m_{n}\right)\right\|^{2}+E\left\|A_{n}\left(\hat{A}_{n}-A_{n}\right) g\right\|^{2}\right] \\
& =O\left(\rho_{n}^{2}\right)\left[E\left\|A_{n}\left(\hat{m}-m_{n}\right)\right\|^{2}+E\left\|A_{n}\left(\hat{A}_{n}-A_{n}\right) g\right\|^{2}\right] .
\end{aligned}
$$

Note that

$$
A_{n}\left(\hat{m}-m_{n}\right)=\sum_{j=1}^{J_{n}}\left(\hat{a}_{j}-a_{j}\right) \psi_{j} .
$$

Define

$$
\tau_{j k}:=E Y^{2} \psi_{j}(W) \psi_{k}(W)-a_{j} a_{k} .
$$


Note that $\tau_{j k}$ is bounded uniformly over $(j, k)$ since $E\left(Y^{2} \mid W=w\right)$ is bounded. Then

$$
\begin{aligned}
E\left\|A_{n}\left(\hat{m}-m_{n}\right)\right\|^{2} & \left.=E \sum_{j=1}^{J_{n}} \sum_{k=1}^{J_{n}}\left\langle\left(\hat{a}_{j}-a_{j}\right) \psi_{j}, \hat{a}_{k}-a_{k}\right) \psi_{k}\right\rangle \\
& =E \sum_{j=1}^{J_{n}}\left(\hat{a}_{j}-a_{j}\right)^{2} \\
& =n^{-1} \sum_{j=1}^{J_{n}} \tau_{j j} \\
& =O\left(\frac{J_{n}}{n}\right) .
\end{aligned}
$$

Now note that

$$
A_{n}\left(\hat{A}_{n}-A_{n}\right) g=\sum_{j=1}^{J_{n}} \sum_{k=1}^{J_{n}} b_{j}\left(\hat{c}_{j k}-c_{j k}\right) \psi_{k} .
$$

Define

$$
\tilde{\tau}_{j k l m}:=E\left[\left\{\psi_{j}(X) \psi_{k}(W)-c_{j k}\right\}\left\{\psi_{l}(X) \psi_{m}(W)-c_{l m}\right\}\right] .
$$

Since $\tilde{\tau}_{j k l m}$ is uniformly bounded over $(j, k, l, m)$, we have that

$$
\begin{aligned}
E\left\|A_{n}\left(\hat{A}_{n}-A_{n}\right) g\right\|^{2} & =E \sum_{j=1}^{J_{n}} \sum_{k=1}^{J_{n}} \sum_{l=1}^{J_{n}} \sum_{m=1}^{J_{n}}\left\langle b_{j}\left(\hat{c}_{j k}-c_{j k}\right) \psi_{k}, b_{l}\left(\hat{c}_{l m}-c_{l m}\right) \psi_{m}\right\rangle \\
& =E \sum_{j=1}^{J_{n}} \sum_{k=1}^{J_{n}} \sum_{l=1}^{J_{n}} b_{j} b_{l}\left(\hat{c}_{j k}-c_{j k}\right)\left(\hat{c}_{l k}-c_{l k}\right) \\
& =n^{-1} \sum_{j=1}^{J_{n}} \sum_{k=1}^{J_{n}} \sum_{l=1}^{J_{n}} b_{j} b_{l} \tilde{\tau}_{j k l k} \\
& \leq\left(J_{n} / n\right)\left[\sum_{j=1}^{J_{n}}\left|b_{j}\right|\right]^{2} \\
& \leq\left(J_{n} / n\right) \sum_{j=1}^{J_{n}} b_{j}^{2} \\
& =O\left(\frac{J_{n}}{n}\right) .
\end{aligned}
$$


It follows that

$$
\left\|\sigma_{n}\right\|^{2}=O\left(\frac{\rho_{n}^{2} J_{n}}{n}\right)
$$

Therefore,

$$
\sigma_{g}(x)=O\left[\rho_{n}\left(\frac{J_{n}}{n}\right)^{1 / 2}\right]
$$

except, possibly, on a set of $x$ 's whose Lebesgue measure is 0 . Thus, we have proved the theorem.

We will first prove Theorem 5.1 and then Theorem 3.2 .

Proof of Theorem 5.1. Define

$$
\Lambda_{n}(x, X, W):=-\left[\hat{g}_{n}(X)-g_{n}(X)\right] \sum_{k=1}^{J_{n}} \psi_{k}(W) \psi_{k}(x)
$$

and

$$
\bar{\Lambda}_{n}(x):=n^{-1} \sum_{i=1}^{n} \Lambda_{n}\left(x, X_{i}, W_{i}\right)
$$

Now write

$$
\tilde{\delta}_{n}^{*}(x, Y, X, W)=\tilde{\delta}_{n}+\Lambda_{n}(x, X, W)
$$

Define $\Delta_{n}:=\hat{A}_{n}-A_{n}$. Then using the fact that

$$
\hat{A}_{n}^{-1}-A_{n}^{-1}=\left[\left(I+A_{n}^{-1} \Delta_{n}\right)^{-1}-I\right] A_{n}^{-1},
$$

we have that

$$
\tilde{S}_{n}^{*}(x)-\hat{A}_{n}^{-1} \bar{\delta}_{n}^{*}(x)=\sum_{l=1}^{4} \tilde{S}_{n l}^{*}(x),
$$

where

$$
\begin{aligned}
& \tilde{S}_{n 1}^{*}(x)=n^{-1} A_{n}^{-1} \sum_{i=1}^{n}\left[\delta_{n}\left(x, Y_{i}^{*}, X_{i}^{*}, W_{i}^{*}\right)-\bar{\delta}_{n}(x)\right], \\
& \tilde{S}_{n 2}^{*}(x)=n^{-1}\left[\left(I+A_{n}^{-1} \Delta_{n}\right)^{-1}-I\right] A_{n}^{-1} \sum_{i=1}^{n}\left[\delta_{n}\left(x, Y_{i}^{*}, X_{i}^{*}, W_{i}^{*}\right)-\bar{\delta}_{n}(x)\right], \\
& \tilde{S}_{n 3}^{*}(x)=n^{-1} A_{n}^{-1} \sum_{i=1}^{n}\left[\Lambda_{n}\left(x, X_{i}^{*}, W_{i}^{*}\right)-\bar{\Lambda}_{n}(x)\right],
\end{aligned}
$$


and

$$
\tilde{S}_{n 4}^{*}(x)=n^{-1}\left[\left(I+A_{n}^{-1} \Delta_{n}\right)^{-1}-I\right] A_{n}^{-1} \sum_{i=1}^{n}\left[\Lambda_{n}\left(x, X_{i}^{*}, W_{i}^{*}\right)-\bar{\Lambda}_{n}(x)\right] .
$$

First, $\tilde{S}_{n 1}^{*}(x)$ is a bootstrap analog of $\tilde{S}_{n}$, so consistency of the bootstrap distribution of $\tilde{S}_{n 1}^{*}(x) / s_{n}(x)$ for that of $\tilde{S}_{n} / s_{n}(x)$ follows immediately from Theorem 1.1 of Mammen (1992). Similarly, the bootstrap distribution of $\sum_{l=1}^{L} \gamma_{l} \tilde{S}_{n 1}^{*}\left(x_{l}\right) / s_{n}\left(x_{l}\right)$ is consistent for that of $\sum_{l=1}^{L} \gamma_{l} \tilde{S}_{n}\left(x_{l}\right) / s_{n}\left(x_{l}\right)$ for any real constants $\gamma_{1}, \ldots, \gamma_{L}$.

Now consider $\tilde{S}_{n 2}^{*}$. Note that

$$
\left\|A_{n}^{-1} \Delta_{n}\right\| \leq\left\|A_{n}^{-1}\right\|\left\|\Delta_{n}\right\|=O_{p}\left[\rho_{n}\left(J_{n} / n\right)^{1 / 2}\right]=o_{p}(1) .
$$

Therefore,

$$
\left\|\left(I+A_{n}^{-1} \Delta_{n}\right)^{-1}-I\right\|=o_{p}(1) .
$$

Since

$$
\tilde{S}_{n 2}^{*}(x)=\left[\left(I+A_{n}^{-1} \Delta_{n}\right)^{-1}-I\right] \tilde{S}_{n 1}^{*}(x),
$$

A.9 implies that

$$
\left\|\tilde{S}_{n 2}^{*}\right\|=o_{p}(1)\left\|\tilde{S}_{n 1}^{*}\right\| .
$$

Now consider $\tilde{S}_{n 3}^{*}$ and $\tilde{S}_{n 4}^{*}$. We have that

$$
\tilde{S}_{n 4}^{*}(x)=\left[\left(I+A_{n}^{-1} \Delta_{n}\right)^{-1}-I\right] \tilde{S}_{n 3}^{*}(x) .
$$

Therefore, again A.9 implies that

$$
\left\|\tilde{S}_{n 4}^{*}\right\|=o_{p}(1)\left\|\tilde{S}_{n 3}^{*}\right\| .
$$

It now suffices to show that $\tilde{S}_{n 3}^{*}$ is asymptotically negligible. To do this, define

$$
\begin{aligned}
\nu_{n}(X) & :=\hat{g}_{n}(X)-g_{n}(X) \\
Z_{n}(W, x) & :=A_{n}^{-1} \sum_{k=1}^{J_{n}} \psi_{k}(W) \psi_{k}(x) .
\end{aligned}
$$

Then

$$
\tilde{S}_{n 3}^{*}(x)=n^{-1} \sum_{i=1}^{n} \nu_{n}\left(X_{i}^{*}\right) Z_{n}\left(W_{i}^{*}, x\right)-n^{-1} \sum_{i=1}^{n} \nu_{n}\left(X_{i}\right) Z_{n}\left(W_{i}, x\right) .
$$


Let $V^{*}$ and $E^{*}$, respectively, denote the variance and expectation relative to the distribution induced by bootstrap sampling. Then $E^{*} \tilde{S}_{n 3}^{*}(x)=$ 0 . Define $V_{n}^{*}(x):=V^{*}\left[\tilde{S}_{n 3}^{*}(x)\right]$. Now note that

$$
\begin{aligned}
V_{n}^{*}(x) & \leq E^{*} n^{-2} \sum_{i=1}^{n} \nu_{n}^{*}\left(X_{i}^{*}\right)^{2} Z_{n}^{*}\left(W_{i}^{*}, x\right)^{2} \\
& =n^{-2} \sum_{i=1}^{n} \nu_{n}^{*}\left(X_{i}\right)^{2} Z_{n}^{*}\left(W_{i}, x\right)^{2} .
\end{aligned}
$$

But, $\nu_{n}\left(X_{i}\right)^{2}=O\left(\left\|\hat{g}_{n}-g_{n}\right\|^{2}\right)=O\left(\left\|\hat{g}_{n}-g\right\|^{2}\right)$ with probability 1 . Therefore,

$$
V_{n}^{*}(x) \leq n^{-2} O\left(\left\|\hat{g}_{n}-g\right\|^{2}\right) \sum_{i=1}^{n} Z_{n}^{*}\left(W_{i}, x\right)^{2}
$$

with probability 1 . Now,

$$
\begin{aligned}
n^{-2} \sum_{i=1}^{n} Z_{n}^{*}\left(W_{i}, x\right)^{2} & =n^{-2} \sum_{i=1}^{n}\left[\sum_{k=1}^{J_{n}} \psi_{k}\left(W_{i}\right)\left(A_{n}^{-1} \psi_{k}\right)(x)\right]^{2} \\
& \equiv R_{n}(x) .
\end{aligned}
$$

But, $\left\|A_{n}^{-1} \psi_{k}\right\| \leq \rho_{n}$, so $\left(A_{n}^{-1} \psi_{k}\right)(x)=O\left(\rho_{n}\right)$ for almost every $x$. Therefore,

$$
\begin{aligned}
R_{n}(x) & \leq O\left(\rho_{n}^{2}\right) n^{-2} \sum_{i=1}^{n}\left[\sum_{k=1}^{J_{n}}\left|\psi_{k}\left(X_{i}\right)\right|\right]^{2} \\
& =O_{p}\left(\frac{\rho_{n}^{2} J_{n}^{2}}{n}\right)
\end{aligned}
$$

by Markov's inequality for almost every $x$. Under Assumption 3.3 . $R_{n}(x)=o_{p}(1)$ for almost every $x$. It follows that for almost every $x$,

$$
V_{n}^{*}(x)=o_{p}\left(\left\|\hat{g}_{n}-g\right\|^{2}\right) .
$$

This combined with the fact that $E^{*} \tilde{S}_{n 3}^{*}(x)=0$ implies that $\tilde{S}_{n 3}^{*}(x)$ is asymptotically negligible for almost every $x$ under sampling from the bootstrap distribution.

Now note that the estimator $s_{n}(x)$ is consistent for $\sigma_{n}(x)$ by Theorem 3.2. Therefore, the first conclusion (5.6) of Theorem 5.1 follows from consistency of the bootstrap distribution of the bootstrap distribution of $\sum_{l=1}^{L} \gamma_{l} \tilde{S}_{n 1}^{*}\left(x_{l}\right) / s_{n}\left(x_{l}\right)$ for that of $\sum_{l=1}^{L} \gamma_{l} \tilde{S}_{n}\left(x_{l}\right) / s_{n}\left(x_{l}\right)$ and the Cramér-Wold device. 
Similarly, the second conclusion (5.7) of Theorem 5.1 follows if we show that $s_{n}^{*}(x)$ is consistent for $\sigma_{n}(x)$, which is proved in Lemma A.4 below.

Proof of Theorem 3.2. Note that we can write $s_{n}^{2}(x)$ as

$$
s_{n}^{2}(x)=n^{-2} \sum_{i=1}^{n}\left\{\hat{A}_{n}^{-1} \delta_{n}^{*}\left(x, Y_{i}, X_{i}, W_{i}\right)\right\}^{2}-n^{-1}\left[\hat{A}_{n}^{-1} \bar{\delta}_{n}^{*}(x)\right]^{2} .
$$

By the arguments used for $\tilde{S}_{n 2}^{*}$ in the proof of Theorem 5.1, replacing $\hat{A}_{n}$ with $A_{n}$ creates an asymptotically negligible error for almost every $x$, it suffices to prove the consistency of

$$
n^{-2} \sum_{i=1}^{n}\left\{A_{n}^{-1} \delta_{n}^{*}\left(x, Y_{i}, X_{i}, W_{i}\right)\right\}^{2}-n^{-1}\left[A_{n}^{-1} \bar{\delta}_{n}^{*}(x)\right]^{2} .
$$

Now

$$
A_{n}^{-1} \tilde{\delta}_{n}^{*}(x, Y, X, W)=A_{n}^{-1} \delta_{n}^{*}(x, Y, X, W)+A_{n}^{-1} \Lambda_{n}(x, X, W) .
$$

Then the second term on the right-hand side of (A.10) is asymptotically negligible for almost every $x$ by the arguments used with $\tilde{S}_{n 3}^{*}$ in the proof of Theorem 5.1. Therefore, it suffices to show that

$$
\sigma_{n}^{-2}(x)\left\{n^{-2} \sum_{i=1}^{n}\left[A_{n}^{-1} \tilde{\delta}_{n}\left(x, Y_{i}, X_{i}, W_{i}\right)\right]^{2}-n^{-1}\left[A_{n}^{-1} \bar{\delta}_{n}(x)\right]^{2}\right\} \rightarrow_{p} 1 .
$$

Note that $\left\{\tilde{\delta}_{n}\left(x, Y_{i}, X_{i}, W_{i}\right)\right\}$ is uniformly integrable by assumption. Then A.11 follows from a triangular-array version of the weak law of large numbers, e.g. Theorem 2 of Andrews (1988).

Lemma A.4. Let Assumptions 3.1 3.6 hold. Then as $n \rightarrow \infty$,

$$
\frac{\left[s_{n}^{*}(x)\right]^{2}}{\sigma_{n}^{2}(x)} \rightarrow p 1
$$

conditional on the original observations $\left\{\left(Y_{i}, X_{i}, W_{i}\right): i=1, \ldots, n\right\}$.

Proof of Lemma A.4. The estimator $\left[s_{n}^{*}(x)\right]^{2}$ differs from $s_{n}^{2}(x)$ by replacing $\hat{g}_{n}$ with $\hat{g}_{n}^{*}, \hat{A}_{n}^{-1}$ with $\left(\hat{A}_{n}^{*}\right)^{-1}$, and $\left\{Y_{i}, X_{i}, W_{i}\right\}$ with $\left\{Y_{i}^{*}, X_{i}^{*}, W_{i}^{*}\right\}$.

Define $\Delta_{n}^{*}:=\hat{A}_{n}^{*}-\hat{A}_{n}$. Then

$$
\left(\hat{A}_{n}^{*}\right)^{-1}-\hat{A}_{n}^{-1}=\left[\left(I+\hat{A}_{n}^{-1} \Delta_{n}^{*}\right)^{-1}-I\right] \hat{A}_{n}^{-1} .
$$


Now using A.7), write

$$
\begin{aligned}
\hat{A}_{n}^{-1} \Delta_{n}^{*} & =A_{n}^{-1} \Delta_{n}^{*}+\left[\hat{A}_{n}^{-1}-A_{n}^{-1}\right] \Delta_{n}^{*} \\
& =A_{n}^{-1} \Delta_{n}^{*}+\left[\left(I+A_{n}^{-1} \Delta_{n}\right)^{-1}-I\right] A_{n}^{-1} \Delta_{n}^{*} .
\end{aligned}
$$

Thus, by A.9),

$$
\left\|\hat{A}_{n}^{-1} \Delta_{n}^{*}\right\| \leq\left[1+o_{p}(1)\right]\left\|A_{n}^{-1} \Delta_{n}^{*}\right\| .
$$

Now as in A.8,

$$
\left\|A_{n}^{-1} \Delta_{n}^{*}\right\| \leq\left\|A_{n}^{-1}\right\|\left\|\Delta_{n}^{*}\right\|=O_{p^{*}}\left[\rho_{n}\left(J_{n} / n\right)^{1 / 2}\right]=o_{p^{*}}(1),
$$

where $p^{*}$ denotes bootstrap probability. It follows from A.12)-(A.14 that

$$
\left\|\left[\left(\hat{A}_{n}^{*}\right)^{-1}-\hat{A}_{n}^{-1}\right] h\right\|=o_{p^{*}}(1)\left\|\hat{A}_{n}^{-1} h\right\|
$$

for any $h \in L_{2}[0,1]$. Therefore, $s_{n}^{*}(x)^{2}$ is asymptotically equivalent to

$$
s_{n 1}^{*}(x)^{2}:=n^{-2} \sum_{i=1}^{n}\left\{\left(\hat{A}_{n}\right)^{-1}\left[\tilde{\delta}_{n}^{* *}\left(x, Y_{i}^{*}, X_{i}^{*}, W_{i}^{*}\right)-\bar{\delta}_{n}^{* *}(x)\right]\right\}^{2} .
$$

Now define $\hat{m}^{*}=\sum_{k=1}^{J_{n}} a_{k}^{*} \psi_{k}$, where $a_{k}^{*}=n^{-1} \sum_{i=1}^{n} Y_{i}^{*} \psi_{k}\left(W_{i}^{*}\right)$. Set

$$
\hat{g}_{n}^{*}=\left(\hat{A}_{n}^{*}\right)^{-1} \hat{m}^{*} \text {. }
$$

Note that this is not the same as 2.4 with the bootstrap sample. Recall that $\hat{h} \equiv \hat{A}_{n}^{-1} \hat{m}$ is asymptotically equivalent to $\hat{g}_{n}$. Then

$$
\begin{aligned}
& \hat{g}_{n}^{*}-\hat{h} \\
& =\left[\left(A_{n}^{*}\right)^{-1}-\hat{A}_{n}^{-1}\right] \hat{m}+\left[\left(A_{n}^{*}\right)^{-1}-\hat{A}_{n}^{-1}\right]\left(\hat{m}^{*}-\hat{m}\right]+\hat{A}_{n}^{-1}\left(\hat{m}^{*}-\hat{m}\right) .
\end{aligned}
$$

Therefore, it follows from $\mathrm{A} .15)$ and the fact that $\left\|\hat{m}^{*}-\hat{m}\right\|=O_{p^{*}}\left[\left(J_{n} / n\right)^{1 / 2}\right]$ that

$$
\left\|\hat{g}_{n}^{*}-\hat{h}\right\|=O_{p^{*}}\left[\rho_{n}\left(J_{n} / n\right)^{1 / 2}\right] .
$$

Consequently, $s_{n}^{*}(x)^{2}$ is asymptotically equivalent to

$$
s_{n 2}^{*}(x)^{2}:=n^{-2} \sum_{i=1}^{n}\left\{\left(\hat{A}_{n}\right)^{-1}\left[\tilde{\delta}_{n}^{*}\left(x, Y_{i}^{*}, X_{i}^{*}, W_{i}^{*}\right)-\bar{\delta}_{n}^{*}(x)\right]\right\}^{2} .
$$

where $\tilde{\delta}_{n}^{*}(x, Y, X, W)$ and $\bar{\delta}_{n}^{*}(x)$ are defined in (3.5) and (3.7), respectively. Then the lemma follows from the consistency of the bootstrap estimator of a sample average. 
TABle 1. Results of Monte Carlo experiments with bootstrap critical values $(\alpha=1.2)$

\begin{tabular}{cccccccc}
\hline $\begin{array}{c}\text { Range } \\
\text { of } x:\end{array}$ & \multicolumn{6}{c}{ Joint Confidence Intervals } & \multicolumn{3}{c}{ Uniform Confidence Band } \\
{$[a, b]$} & $J_{n}$ & 0.90 & 0.95 & 0.99 & 0.90 & 0.95 & 0.99 \\
\hline \multirow{6}{c}{ Bootstrap Critical Values I } \\
$(0.2,0.8)$ & 3 & 0.866 & 0.923 & 0.962 & 0.872 & 0.926 & 0.962 \\
& 4 & 0.913 & 0.953 & 0.986 & 0.920 & 0.957 & 0.986 \\
& 5 & 0.929 & 0.962 & 0.987 & 0.935 & 0.965 & 0.989 \\
& 6 & 0.933 & 0.966 & 0.989 & 0.938 & 0.970 & 0.990 \\
$(0.1,0.9)$ & 3 & 0.851 & 0.893 & 0.944 & 0.859 & 0.904 & 0.948 \\
& 4 & 0.826 & 0.883 & 0.926 & 0.838 & 0.886 & 0.931 \\
& 5 & 0.874 & 0.914 & 0.963 & 0.883 & 0.921 & 0.964 \\
& 6 & 0.896 & 0.940 & 0.975 & 0.903 & 0.947 & 0.979 \\
$(0.01,0.99)$ & 3 & 0.848 & 0.896 & 0.945 & 0.862 & 0.906 & 0.952 \\
& 4 & 0.808 & 0.864 & 0.921 & 0.830 & 0.870 & 0.929 \\
& 5 & 0.790 & 0.856 & 0.919 & 0.817 & 0.874 & 0.934 \\
& 6 & 0.788 & 0.849 & 0.916 & 0.825 & 0.873 & 0.937 \\
\hline \multirow{3}{*}{$(0.2,0.8)$} & 3 & 0.911 & 0.951 & 0.981 & 0.914 & 0.951 & 0.981 \\
& 4 & 0.929 & 0.968 & 0.992 & 0.935 & 0.971 & 0.992 \\
& 5 & 0.948 & 0.981 & 0.997 & 0.953 & 0.984 & 0.997 \\
& 6 & 0.955 & 0.987 & 0.997 & 0.959 & 0.989 & 0.997 \\
& 3 & 0.907 & 0.946 & 0.989 & 0.912 & 0.949 & 0.991 \\
& 4 & 0.904 & 0.938 & 0.986 & 0.907 & 0.940 & 0.988 \\
& 5 & 0.926 & 0.966 & 0.991 & 0.932 & 0.967 & 0.991 \\
& 6 & 0.949 & 0.980 & 0.997 & 0.956 & 0.982 & 0.997 \\
& 3 & 0.905 & 0.946 & 0.989 & 0.911 & 0.955 & 0.993 \\
& 4 & 0.895 & 0.949 & 0.992 & 0.910 & 0.957 & 0.993 \\
& 5 & 0.922 & 0.964 & 0.995 & 0.931 & 0.973 & 0.997 \\
& 6 & 0.943 & 0.976 & 0.996 & 0.957 & 0.984 & 0.997 \\
\hline $0.1,0.9)$ & & $70.99)$ & & & & &
\end{tabular}

Note: This table shows coverage probabilities of the joint confidence intervals and uniform confidence band for $g(x)$. Two types of bootstrap critical values are considered: $t_{n}^{*}(x)$ in (5.1) (bootstrap critical value I) and $t_{n}^{* *}(x)$ in (5.2) (bootstrap critical value II). 
TABle 2. Results of Monte Carlo experiments with bootstrap critical values $(\alpha=10)$

\begin{tabular}{cccccccc}
\hline $\begin{array}{c}\text { Range } \\
\text { of } x:\end{array}$ & \multicolumn{6}{c}{ Joint Confidence Intervals } & \multicolumn{3}{c}{ Uniform Confidence Band } \\
{$[a, b]$} & $J_{n}$ & 0.90 & 0.95 & 0.99 & 0.90 & 0.95 & 0.99 \\
\hline \multirow{6}{c}{ Bootstrap Critical Values I } \\
$(0.2,0.8)$ & 3 & 0.656 & 0.701 & 0.768 & 0.659 & 0.702 & 0.770 \\
& 4 & 0.727 & 0.770 & 0.846 & 0.738 & 0.778 & 0.848 \\
& 5 & 0.745 & 0.793 & 0.871 & 0.749 & 0.800 & 0.877 \\
& 6 & 0.776 & 0.821 & 0.890 & 0.789 & 0.831 & 0.897 \\
$(0.1,0.9)$ & 3 & 0.652 & 0.699 & 0.765 & 0.660 & 0.702 & 0.768 \\
& 4 & 0.695 & 0.736 & 0.812 & 0.702 & 0.743 & 0.820 \\
& 5 & 0.699 & 0.755 & 0.829 & 0.710 & 0.765 & 0.843 \\
& 6 & 0.742 & 0.790 & 0.867 & 0.766 & 0.808 & 0.875 \\
$(0.01,0.99)$ & 3 & 0.649 & 0.700 & 0.765 & 0.661 & 0.704 & 0.768 \\
& 4 & 0.692 & 0.732 & 0.811 & 0.708 & 0.745 & 0.819 \\
& 5 & 0.699 & 0.749 & 0.831 & 0.720 & 0.765 & 0.846 \\
& 6 & 0.745 & 0.793 & 0.865 & 0.773 & 0.820 & 0.882 \\
\hline \multirow{3}{*}{$(0.2,0.8)$} & 3 & 0.891 & 0.938 & 0.975 & 0.894 & 0.939 & 0.976 \\
& 4 & 0.915 & 0.948 & 0.983 & 0.915 & 0.950 & 0.983 \\
& 5 & 0.930 & 0.970 & 0.991 & 0.931 & 0.970 & 0.991 \\
& 6 & 0.960 & 0.977 & 0.995 & 0.961 & 0.980 & 0.995 \\
& 3 & 0.892 & 0.940 & 0.979 & 0.893 & 0.940 & 0.979 \\
& 4 & 0.915 & 0.954 & 0.986 & 0.917 & 0.955 & 0.986 \\
& 5 & 0.936 & 0.970 & 0.991 & 0.937 & 0.971 & 0.991 \\
& 6 & 0.955 & 0.979 & 0.996 & 0.956 & 0.979 & 0.996 \\
& 3 & 0.892 & 0.942 & 0.979 & 0.894 & 0.944 & 0.979 \\
& 4 & 0.917 & 0.959 & 0.986 & 0.923 & 0.960 & 0.986 \\
& 5 & 0.940 & 0.973 & 0.993 & 0.943 & 0.973 & 0.993 \\
& 6 & 0.962 & 0.984 & 1.000 & 0.965 & 0.984 & 1.000 \\
\hline $0.1,0.9)$ & & $70.99)$ & & & & &
\end{tabular}

Note: This table shows coverage probabilities of the joint confidence intervals and uniform confidence band for $g(x)$. Two types of bootstrap critical values are considered: $t_{n}^{*}(x)$ in (5.1) (bootstrap critical value I) and $t_{n}^{* *}(x)$ in (5.2) (bootstrap critical value II). 


\section{REFERENCES}

Andrews, D. W. K. (1988): Laws of Large Numbers for Dependent Non-Identically Distributed Random Variables, Econometric Theory, 4, 458-467.

Blundell, R., X. Chen, and D. Kristensen (2007): Semi-nonparametric IV estimation of shape-invariant Engel curves, Econometrica, 75, 16131669.

Chen, X. and D. Pouzo (2008): Estimation of nonparametric conditional moment models with possibly nonsmooth moments, Cemmap Working Papers, CWP12/08, available at: http://cemmap.ifs.org.uk.

Chen, X. and D. Pouzo (2009): Efficient estimation of semiparametric conditional moment models with possibly nonsmooth residuals, Journal of Econometrics, forthcoming.

Chernozhukov, V, G. W. Imbens and W. K. Newey (2007): Instrumental variable estimation of nonseparable models, Journal of Econometrics, 139, 4-14.

Chernozhukov, V., P. Gagliardini, and O. Scaillet (2008): Nonparametric instrumental variable estimation of quantile structural effects, working paper, Department of Economics, Massachusetts Institute of Technology, Cambridge, MA.

Darolles, S., J. -P. Florens, and E. Renault (2006): Nonparametric instrumental regression, Working paper, GREMAQ, University of Social Science, Toulouse, France.

Hall, P. and J. L. Howowitz (2005): Nonparametric methods for inference in the presence of instrumental variables, Annals of Statistics, 33, 2904-2929.

Hall, P. and D. M. Titterington (1988): On confidence bands in nonparametric density estimation and regression, Journal of Multivariate Analysis, 27, 228-254.

Horowitz, J. L. (2007): Asymptotic Normality of a Nonparametric Instrumental Variables Estimator, International Economic Review, 48, 1329-1349. 
Horowitz, J. L. (2008): Specification Testing in Nonparametric Instrumental Variables Estimation, working paper, Department of Economics, Northwestern University, Evanston, IL.

Horowitz, J. L. and S. Lee (2007): Nonparametric instrumental variables estimation of a quantile regression model, Econometrica, 75, 1191-1208.

Mammen, E. (1992). When Does Bootstrap Work? Asymptotic Results and Simulations, New York: Springer-Verlag.

Newey, W. K., J. L. Powell, and F. Vella (1999): Nonparametric estimation of triangular simultaneous models, Econometrica, 67, 565-603.

Wang, J. and L. Yang (2009). Polynomial spline confidence bands for regression curves, Statistica Sinica, 19, 325-342.

Zhou, S., X. Shen, and D.A. Wolfe (1998). Local asymptotics of regression splines and confidence regions, Annals of Statistics, 26, 1760-1782.

Department of ECONOMICS,NORTHWESTERn UNIVERSity AND DEPARTMENT of ECONOMICS, UNIVERSITY COLLEGE LONDON

E-mail address: joel-horowitz@northwestern.edu and 1.simon@ucl.ac.uk 